\title{
A Mobility Control Architecture Supporting Fast Handover for Future Internet Networks
}

\author{
Khanh-Huy Nguyen ${ }^{\dagger}$, Won-Joo Hwang ${ }^{\dagger+}$
}

\begin{abstract}
In this paper, we address the problem of fast handover support for future internet networks. The current mobility support protocols, for example Proxy Mobile IPv6, follow a centralized architecture in which a mobility controller is used to manage intra-network handovers that gives rise to high handover latency. To handle this problem, we propose a novel distributed mobility control architecture in which gateways in the network can exchange user information with neighbour gateways to support intra-network handovers, the mobility controller is only needed in case of inter-network handovers. Simulation results show that our architecture reduce approximately 20\% intra-network handover latency than Proxy Mobile IPv6.
\end{abstract}

Key words: Mobility Control, Future Internet, Handover Latency, Intra-network Handover, Internetwork Handover

\section{INTRODUCTION}

Future internet networks are focused on developing all-IP mobile networks. One of the most important challenges for future all-IP mobile networks is mobility management within fast handover support to limit handover latency of users. Almost current protocols for internet mobile networks are based on centralized architectures in which mobility management and handover procedures are processed by a centralized mobility controller, for example Local Mobility Anchor (LMA)

※ Corresponding Author: Won-Joo Hwang, Address: (621-749) Obangdong, Gimhae, Gyeongnam, Korea, TEL : +82-55-320-3847, FAX : +82-55-322-6275, E-mail : ichwang@inje.ac.kr

Receipt date: Nov. 23, 2012, Revision date: Nov. 30, 2012 Approval date: Dec. 7, 2012

${ }^{+}$Dept. of Information and Communications Engineering, UHRC, Inje University

(E-mail: huynkh@gmail.com)

${ }^{+\dagger}$ Dept. of Information and Communications Engineering, UHRC, Inje University

※ This research was supported by Basic Science Research Program through the National Research Foundation of Korea (NRF) funded by the Ministry of Education, Science and Technology (2011-0024888). of Proxy Mobile IPv6 (PMIP) [1] or Home Agent (HA) of Mobile IPv6 (MIP) [2,3]. Each centralized mobility controller manages all user information for a network. Therefore, such controllers are necessary to support inter-network handovers when a user moves from a network to another. However, in case of intra-network handovers when a user moves among sub-networks controled by gateways, transmission handover control information among old gateway-centralized mobility controller-new gateway gives rise to high handover latency.

For purpose of decreasing the handover latency of intra-network handovers, in this paper, we propose a novel distributed mobility control architecture in which gateways in the network can $\mathrm{ex}^{-}$ change user information, i.e. IP address (user ID), with neighbour gateways to support intra-network handovers. In stead of transmission handover control information from the old gateway to the centralized mobility controller and from the centralized mobility controller to the new gateway, gateways in a network exchange directly user information for handover procedure with neighbour gateways. 
Then, the new gateway serving the user is updated to the mobility controller. The centralized mobility controller is only needed in case of inter-network handovers.

The rest of this paper is organized as follows. Section 2 reviews the existing works in design of mobility control architecture for future internet networks. In Section 3, we present the proposed mobility control architecture. Simulation results are shown in Section 4, and Section 5 concludes the paper.

\section{RELATED WORK}

The recent basic future networking trend is focusing on developing all-IP mobile networks within resolving mobility management problems including fast handover support. The authors in [1] introduce about Proxy Mobile IPv6 (PMIP) protocol for internet mobility. In PMIP, they use a centralized mobility anchor, i.e. Local Mobility Anchor (LMA), to process all data and control traffics. Mobility management and fast handover support for Mobile IPv6 (MIP) are provided in [2,3]. the MIP supports mobility of mobile node using tow address such as Home Address (HoA) by its Home Agent (HA) and Care-of-Address (CoA) by a visited network. The HA is the centralized mobility controller in MIP. The authors in [4] provide a review on mobility management for future IP-based next generation wireless networks. This review introduces network structure and characteristic of MIP, PMIP and Hierarchical MIP. In [5], comparison between MIP and PMIP in mobility management for all-IP mobile networks is described. An $\mathrm{ID} /$ locator split architecture for future internet is introduced in [6]. This kind of architecture also uses centralized nodes such as domain name registry, ID registry and host name registry in network to manage mobility procedures of users. In [7], the authors provide a new generation network within discussing internet and next generation networks.
A handover latency analysis of IPv6 mobility management protocols in all-IP networks is provided in [8]. In [9], the authors propose an analysis of the capacity region for a kind of two-tier spatial diversified network. Most of these existing protocols proposed for future internet networks are based on the centralized architectures in which a centralized mobility controller is used to process all data traffics and mobility management for both inter-network handover and intra-network handover. As explain above, that makes high latency problem for handover procedure in case of intra-network handover. To overcome this problem, we propose a novel distributed mobility control architecture in next section, in which gateways in the network can exchange user information with neighbour gateways to support intra-network handovers, the mobility controller is only needed in case of inter-network handovers.

\section{PROPOSED NETWORK ARCHITECTURE}

In this section, we describe the proposed network architecture to support fast handover for intra-network handover. Inter-network handover is also discussed.

\subsection{Architecture Layout}

The layout of our proposed network architecture is shown in Fig. 1. Basically, it includes an internet backbone network, access networks and a logical control network.

The internet backbone network contains backbone routers in order to convey data packets from a source access network to a destination access network. It usually has a stationary configuration.

The access networks supply internet network access to diverse kinds of users. They might be an ad hoc network, a wireless sensor network, a vehicular network and so on, whose topology alters frequently due to mobility of users. The access networks connect to the internet backbone network 


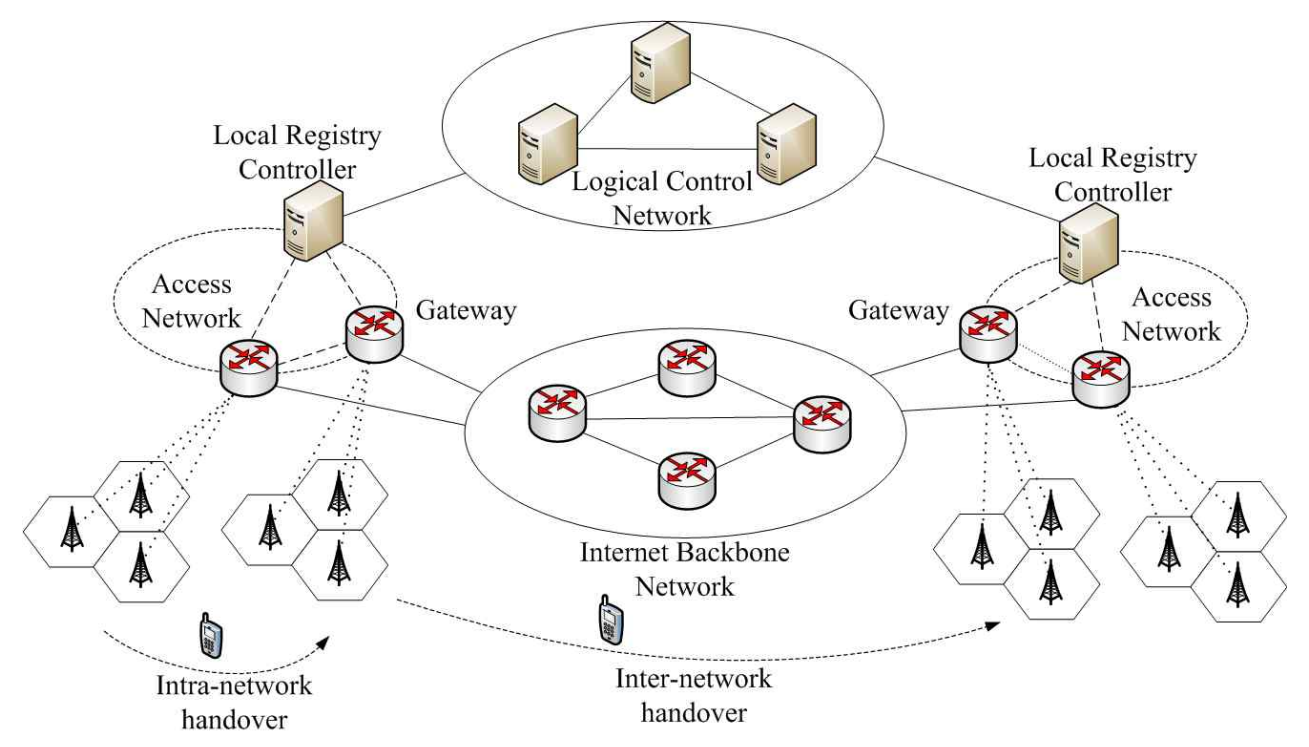

Fig. 1. Architecture layout.

through gateways. The gateways have functions to translate local address into global address for packets passing through them.

The logical control network is the third component of the architecture. It comprises local registry controllers (LRCs). Each access network is managed by a LRC. The LRCs contain information such as authorization, network configuration, QoS management, authentication and user ID (IP address). Similar to LMA in PMIP, when a mobile node joins an access network, it is assigned a unique IP address by the LRC. This IP address is considered as user ID for the mobile node and maintained during the mobile node is joining in the access network even it moves through some gateways. However, being different from LMA of PMIP, while LMA forwards directly IP packets from the source node to the destination node, the LRC is only manage and contain network information. It does not join forwarding packets. This mission is executed only by gateways under management of the LRC. That not only saves time to forward packets but also reduces complexity of the network.

Each gateway manages a subnet within connecting to several cells and contains IP address of all users it is serving. The gateways translate be- tween local ID and global ID for packets passing through them when the source node and destination node are in different networks. There is a novel function of the gateways we propose here. The neighbour gateways in a network can exchange each other the user ID list that they are serving, Then, when a mobile node move from a subnet to another neighbour subnet, the new gateway can fast serve the mobile node, saving handover procedure time. Without waiting time from the LRC to know user $\mathrm{ID}$ in a new subnet, that time reduction is really significant in comparison with PMIP when the neighbour gateways do not know served user ID list each other and always have to connect with LMA to authenticate the mobile node in a new subnet. The LRC is only needed for handover procedure in case the mobile node move from a network to another network.

\subsection{User Registration and Resolution}

When a user joins a network, it firstly must have registered with the $\mathrm{LRC}$ of the network to be authenticated and get its ID in the network. After that, it can communicate with other users in other networks using its assigned ID

Now, we see how two nodes in our proposed 


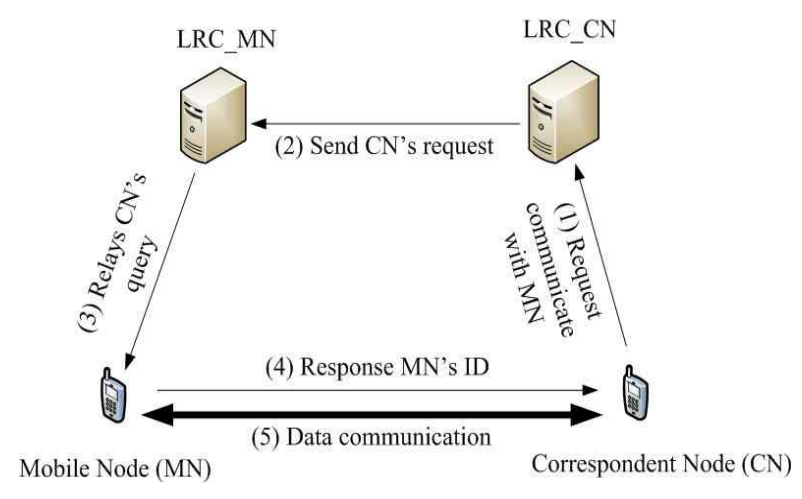

Fig. 2. Communication process.

network can communicate each other. Fig. 2 shows the communications process between a mobile node $(\mathrm{MN})$ and a correspondent node $(\mathrm{CN})$ in another network. The CN wants to communicate with the MN and it gets MN's ID by querying the LRC_CN and LRC_MN. Firstly, the CN sends request communicate with the MN to the LRC_CN. Through the logical control network, the LRC_CN sends this request to the LRC_MN. The LRC_MN then checks its serving user list and relays the query of the $\mathrm{CN}$ to the MN. Next, the MN replies its ID to the $\mathrm{CN}$. Note that, the MN gets the $\mathrm{CN}^{\prime} \mathrm{s}$ ID from the query message of the $\mathrm{CN}$ and the $\mathrm{CN}$ gets the MN's ID from the response message of the MN. After acquiring the IDs each other, the $\mathrm{MN}$ and the $\mathrm{CN}$ can start data communication.

\subsection{Intra-network Fast Handover Support}

The gateways in our proposed network architecture have a novel function comparing to existing network architectures. That is they can exchange serving user ID list with the neighbour gateways. This is significantly useful to reduce handover latency in case of intra-network handovers when the MN moves from a subnet to another neighbour subnet.

Fig. 3 shows intra-network handover procedure when the MN moves from old gateway to new gateway until it can resume communicate with the $\mathrm{CN}$. At first, when the old gateway detects movement of the MN away from its subnet, it informs

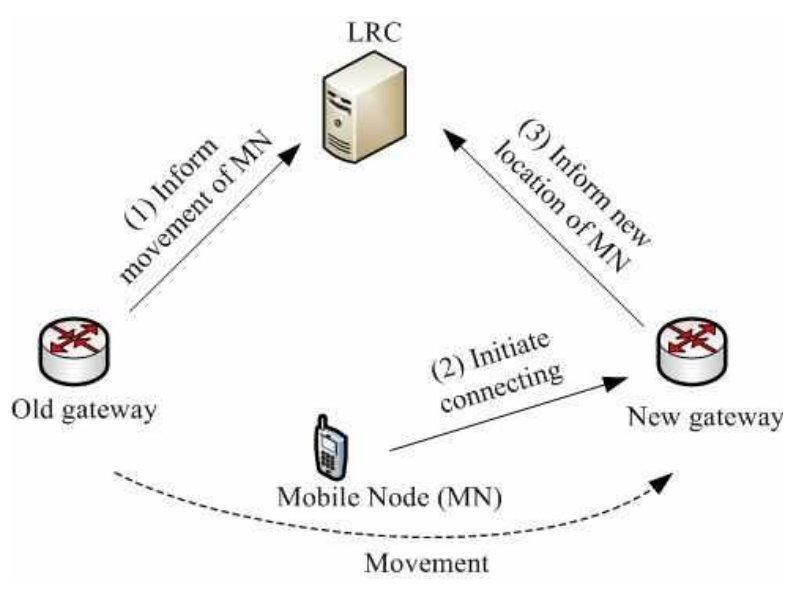

Fig. 3. Intra-network handover process.

that to the LRC by sending a message. The MN initiates connecting to subnet of the new gateway. Because the new gateway already knew about the MN's ID by exchange user information with the old gateway, it can serve the MN immediately without waiting for authentication from the LRC. The new gateway then sends a message to the LRC to inform new location of the MN. After that, the $\mathrm{MN}$ can send/receive packets to/from the $\mathrm{CN}$ through the new gateway.

Exchanging user information with the old gateway, the new gateway can predict movement of the MN and serve it faster in comparison with other existing network architectures. For example, in PMIP, the new gateway needs to wait for authentication of the MN's ID from LMA before serving the MN. This waiting time duration gives rise to higher handover latency. Therefore, our proposed network architecture can reduce handover latency better.

\subsection{Inter-network Mobility Support}

Our proposed architecture also supports internetwork handover in which the MN moves from a network to another network.

Fig. 4 shows the inter-network handover procedure. We suppose that the $\mathrm{MN}$ is communicating with the $\mathrm{CN}$ and then it moves from the old network to the new network. In order to serve com- 


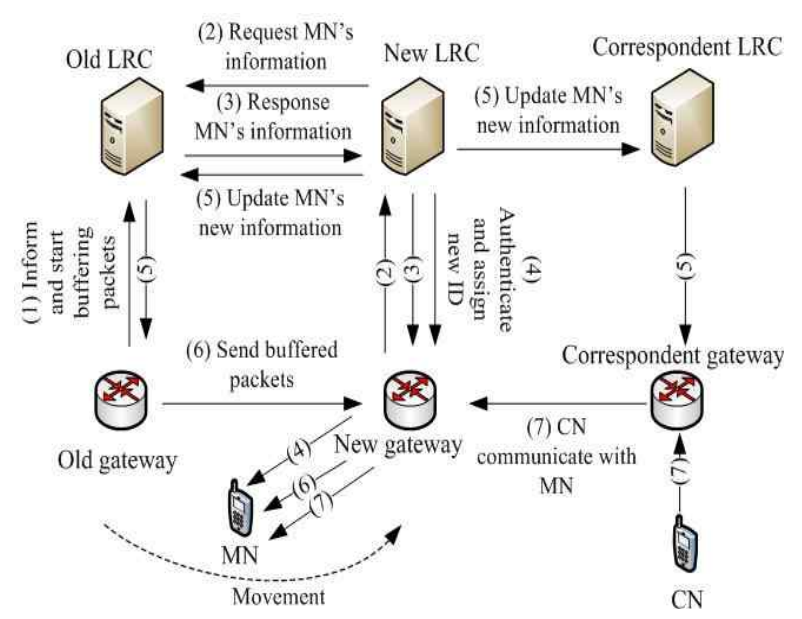

Fig. 4. Inter-network handover process.

munication between the $\mathrm{MN}$ and the $\mathrm{CN}$, the old gateway stores information about IDs of the MN, the correspondent gateway and the CN. Similarly, the correspondent gateway also stores information about IDs of the $\mathrm{CN}$, the old gateway and the MN. This information is also saved in the old LRC and the correspondent LRC. When the old gateway detects movement of the MN to the new network, it informs the LRC and starts buffering data packets from the $\mathrm{CN}$ to be sent to the MN. As soon as the new gateway detects the $\mathrm{MN}$ in its network, it requests the new LRC to get all information of the MN from the old LRC. The old LRC then sends all information of the MN to the new LRC. Alternately, the new LRC authenticates and assigns a new ID for the MN in its network. After that, the new LRC update the MN's new ID and the new gateway's ID to the old LRC and the correspondent LRC. This information next is sent to the old gateway and the correspondent gateway. Then, the old gateway sends the buffered data packets to the MN through the new gateway and the $\mathrm{CN}$ communicates with the $\mathrm{MN}$ using the new ID of the MN.

\section{SIMULATION RESULTS}

To evaluate handover latency in our proposed network architecture, we consider a pico cellular environment with a hexagonal cell. We randomize 10 mobile nodes taking handover in both intra-network and inter-network. The analysis setting and the parameters used for the analysis are based on [8]. We assume latency of an IP packet delivery between MN and gateway latency of IP packet's one node delivery only through wired medium are from $2 \mathrm{~ms}$ to $4 \mathrm{~ms}$ for each handover process. The simulation tool used for the numerical analysis is MATLAB 7.8.0.

We first evaluate handover latency in case of intra-network handovers. Fig. 5 shows comparison in handover latency between our proposed network architecture and PMIP architecture. As shown in Figure 5, our proposed network architecture can obtain the total handover latency approximately 20\% less then PMIP architecture. That is because when the MN moves from a subnet to another subnet, the new gateway needs to inform and waits for authentication from the LMA to enable the MN in the new subnet in PMIP. By contrast, in our proposed architecture, the new gateway can serve the $\mathrm{MN}$ immediately without waiting time for $\mathrm{au}^{-}$ thentication from the LRC when the MN join in its subnet by knowing the MN's ID in advance. Therefore, our proposed network architecture can support handover processes faster.

We also estimate handover latency in case of inter-network handovers. Fig. 6 depicts the handover latency of inter-network handover processes.

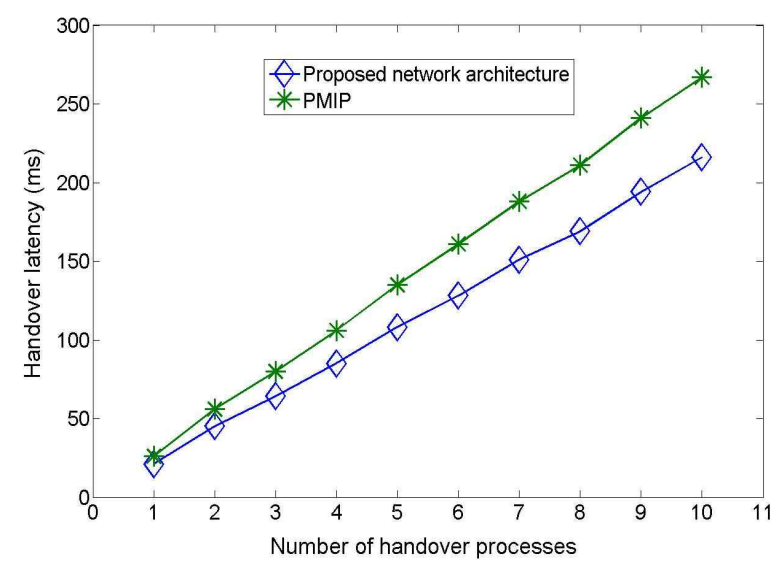

Fig. 5. Intra-network handover latency comparison. 


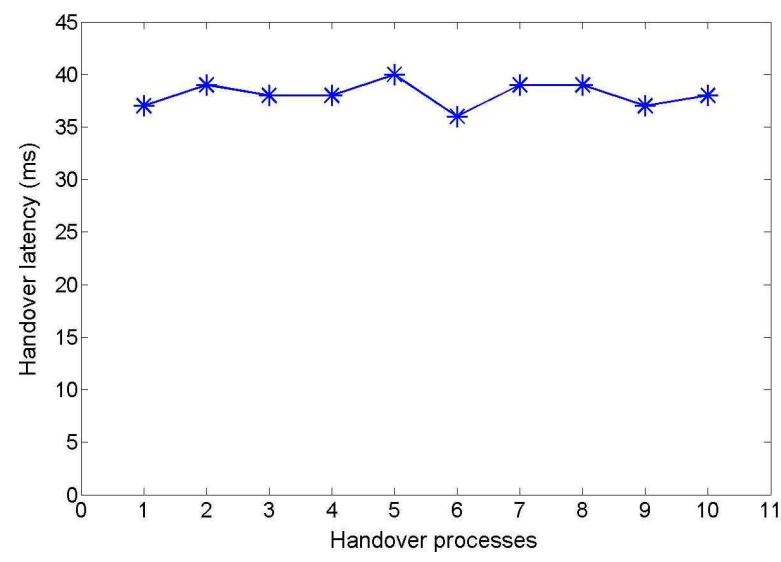

Fig 6. Inter-network handover latency.

The average latency for the network layer handover is obtained from $36 \mathrm{~ms}$ to $40 \mathrm{~ms}$. These values are acceptable in the real networks for handover processes.

\section{CONCLUSION}

In this paper, we consider the problem of fast handover support for future internet networks. In order to decrease handover latency for intra-network handover cases, we propose a novel distributed mobility control architecture in which gateways in the network can exchange user information with neighbour gateways in advance. The mobility controllers in our proposed architecture do not transmit directly packets from source node to destination node. They just control network configuration, user information and only needed in case of inter-network handovers. Simulation results show that our architecture reduce approximately 20\% intra-network handover latency in comparison with Proxy Mobile IPv6.

\section{REFERENCES}

[1] S. Gundavelli, K. Leung, V. Devarapalli, K. Chowdhury, and B. Patil, "Proxy Mobile IPv6," IETF RFC 5213, pp. 1-92, 2008.

[2] D. Johnson, C. Perkins, and J. Arkko, "Mobility Support in IPv6," IETF RFC 3775, pp. 1-165, 2004.

[3 ] R. Koodli, "Fast Handover for Mobile IPv6," IETF RFC 4068, pp. 1-42, 2005.

[4] I. Al-Surmi, M. Othman, and B.M Ali, "Review on Mobility Management for FutureIP-based Next Generation Wireless Networks," Proc. IEEE ICACT, Vol. 2, pp. 989-994, 2010.

[5] K.S. Kong, W.J. Lee, Y.H. Han, M.K. Shin, and H.R. You, "Mobility Management for All-IP Mobile Networks: Mobile IPv6 vs. Proxy Mobile IPv6," IEEE Wireless Communications Magazine, Vol. 15, No. 2, pp. 36-45, 2008.

[6] V.P. Kafle, H. Otsuki, and M. Inoue, "An ID/locator Split Architecture for Future Networks," IEEE Communications Magazine, Vol. 48, No. 2, pp. 138-144, 2010.

[7] T. Aoyama, "A New Generation Network: Beyond the Internet and NGN," IEEE Communications Magazine, Vol. 47, No. 5, pp. 8287, 2009.

[8] D.K. Panwar, S. Lal, and I. Chaudhary, "Handover Latency Analysis of Mobility Management Protocols in All-IP Networks," Proc. IEEE ACT, pp. 390-394, 2009.

[9] J. Torregoza, M. Choi, and W. Hwang, "Analysis of the Capacity Region for Two-tier Spatial Diversified Wireless Mesh Networks," Journal of The Korea Multimedia Society, Vol. 11, No. 12, pp. 1697-1705, 2008. 


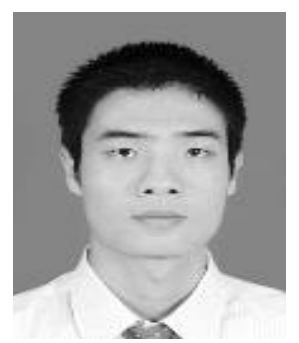

\section{Khanh-Huy Nguyen}

He received his bachelor's degree in Electronic and Telecommunications from Hanoi University of Science and Technology, Vietnam in 2009. He received his M.S. degree in Computer Engineering from Inje University, Gimhae, Republic of Korea in 2012, He is now Ph.D candidate of the Department of Electronic and Telecommunications Engineering, Inje University. His research interests are resource management in cognitive radio network and interference coordination problems in small-cell networks.

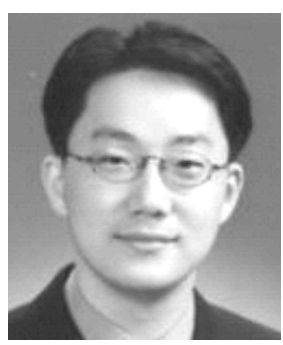

\section{Won-Joo Hwang}

He received the Ph.D Degree from Osaka University Japan in 2002. He received his bachelor's degree and M.S. degree in Computer Engineering from Pusan National University, Pusan, $\mathrm{Re}^{-}$ public of Korea, in 1998 and 2000. Since September 2002, he has been an associate professor at Inje University, Republic of Korea. His research interests are in Network Optimization and Ubiquitous Sensor Networks. 